\title{
Health Risk Assessment Indicators for the Left-Behind Elderly in Rural China: A Delphi Study
}

\author{
Ruzhen Luo, Chunmei Zhang and Yanhui Liu* \\ School of Nursing, Tianjin University of Traditional Chinese Medicine, Tianjin 301617, China; \\ lrztjutcm@163.com (R.L.); suiyue801@163.com (C.Z.) \\ * Correspondence: yh_liu888@163.com; Tel.: +86-022-5979-1931
}

Received: 6 November 2019; Accepted: 1 January 2020; Published: 3 January 2020

\begin{abstract}
In China, many young and middle-aged rural residents move to urban areas each year. The rural elderly are left behind. The number of the rural left-behind elderly is increasing with urbanization, but it is unclear which indicators can be used to assess their health condition. The health risk assessment index system was developed to improve the health level of the rural left-behind elderly. A two-round web-based Delphi process was used to organize the recommendations from fifteen Chinese experts in geriatrics, health management, social psychology who participated in this study. Meaningfulness, importance, modifiability, and comprehensive value of the health risk assessment indicators in the index system were evaluated. The effective recovery rates of the two-round Delphi were $86.67 \%$ and $92.31 \%$, respectively. The judgement coefficient and the authority coefficient were 0.87 and 0.82 , respectively. The expert familiarity was 0.76 . Ultimately, the health risk assessment index system for the rural left-behind elderly consisted of five first-level indicators, thirteen second-level indicators, and sixty-six third-level indicators. The final indicators can be used to evaluate the health of the rural left-behind elderly and provide the basis for additional health risk interventions.
\end{abstract}

Keywords: left-behind elderly; health risk assessment; Delphi technique

\section{Introduction}

Traditionally, Chinese elderly are taken care of by their children. But it becomes more and more difficult with the deepening of urbanization, domestic migration, and deconstruction of extended families. With the rapid development of the social economy, remarkable demographic transitions have taken place in China in the past three decades. Approximately 250 million rural residents $(40 \%$ of the whole rural population) move to urban areas each year, most of them are young and middle-aged migrant workers. Therefore, a large number of rural children, women, and elderly are left behind [1,2]. Left-behind elderly refers to the elderly over 60 who live in rural areas while their children have been away from home six months at least [3]. According to the latest report of the National Bureau of Statistics, there are 127 million elderly people in rural China as of 2014. The number of left-behind elderly in rural areas has grown to 50 million, which account for $39.37 \%$ of the total rural elderly population. By 2020, the number of the elderly over 60 will increase to 255 million, and the number of elderly people living alone will increase to 118 million. The urbanization process and large-scale population migration will continue for a long time in China. By 2020, 300 million rural people at least will enter cities. Therefore, the number of the rural left-behind elderly will continue to increase.

The interaction between health and migration is complex, dynamic, and bidirectional. Migration not only affects the physical, mental, and emotional health and well-being of the migrants themselves, but also affects the health of the left-behind in the place of origin [4]. The elderly often suffer from chronic disease, such as heart disease, stroke, cancer, diabetes, arthritis, and falls [5]. 
Additionally, the challenges to mental health, such as depression, Alzheimer's disease, and dementia, are the common health problems that the elderly often worry about [5]. Moreover, the elderly also face many social challenges besides physical and mental health problems [6].

The health condition of the left-behind elderly in rural areas is generally low in China, which is caused by low children support, high living pressure, low utilization rate of health resources, and incomplete low level of rural pension security in rural areas and so on. Chinese scholars generally believe that health is the most important problem to solve for the left-behind elderly in rural areas [7-9]. A survey of 1811 left-behind elderly in Thailand found that the health of the left-behind elderly in rural Thailand is poor. The results showed that the left-behind elderly in rural areas had a high risk of depression [10]. Antman surveyed 5247 Mexican left-behind elderly and 1483 Mexican non-left-behind elderly. The results showed that the risk of stroke and heart disease is higher in the left-behind elderly than that in the non-left-behind elderly. And the left-behind elderly were more likely to have negative emotions such as anxiety and depression than the non-left-behind elderly [11]. He et al. used the Geriatric Depression Scale to investigate the incidence of depression among 509 rural left-behind elderly in China, the results showed that the incidence of depression in the rural left-behind elderly was $36.49 \%$. And the incidence of depression in women was $45.1 \%$, which was higher than $33.43 \%$ in men [12]. Miltiades found that although the rural elderly obtained economic support from their children, the migration of children would directly affect the psychological status of the rural left-behind elderly; the elderly generally felt lonely and depressed [13].

Previous studies have also focused on the influencing factors of the health status among the left-behind elderly. A study of 1619 elderly people in Germany showed that age was the important influencing factor of mental health. The older the left-behind elderly, the greater the impact on their health [14]. Antman deemed that women, age, and the year of education were related to the physical and mental health of the left-behind elderly [11]. The survey was conducted among 6000 rural left-behind elderly over 60 in Gansu and showed that the prevalence rate of mental illness was $20.11 \%$. Among all kinds of mental diseases, depression (9.20\%), pain disorder (2.71\%), and mood disorder due to physical condition (2.08\%) rank the first three. The prevalence of female $(242.89 \%)$ was significantly higher than that of male $(119.55 \%)$, and the prevalence of unmarried $(248.37 \%)$ was significantly higher than that of married (187.53\%) [15]. Forlani et al. and Houtjes et al. pointed out that the gender, age, and the educational level were related to the physical and mental health of the elderly [16,17]. Williams et al. pointed out that religious beliefs, educational level, marital status, and family support can affect the quality of life of the elderly $[18,19]$. Xie et al. showed that types of chronic diseases, living style, and financial support of children of the left-behind elderly in rural areas are influencing factors of mental health [20]. A study among 509 rural left-behind elderly in China showed that the risks of depression were gender, visiting frequency of children, living environment, physical activity ability, types of chronic diseases, and the education level [12]. A survey among the elderly in Thailand found that, after controlling socio-demographic and economic variables, the elderly who had a migrant child were more likely to have poor mental health $(\mathrm{OR}=1.10$; $95 \% \mathrm{CI} 1.05-1.17)$ than those children who had not migrated [21].

All in all, the prominent health problems not only reduce the quality of life of the rural left-behind elderly, but also increase the care pressure and the financial burden. The expenditure of the national long-term care for the elderly also increased. Present studies mainly focus on the physical and mental health status and its influencing factors of the rural left-behind elderly. There are few studies exploring the health risk assessment indicators for the rural left-behind elderly.

Health ecology model is a theoretical model originated from ecology. It is an important model to solve human health problems in preventive medicine and public health [22]. The model shows that people's health is influenced by individual factors, social environment, medical and health services. The health ecology model includes five layers: the core layer is the personal traits, the second layer is the behavior characteristics, the third layer is the interpersonal network, the fourth layer is the living and working conditions, and the fifth layer is the social, economic, cultural, and related policies. At present, 
it has been applied to the studies of obesity, nutrition, smoking cessation control, self-management of diabetes, and the explanations of healthy influencing factors [23-26]. In the analysis of influencing factors, the health ecology model not only involves individual physiology, psychology, life style, physical environment, and social environment, but also emphasizes the interaction of these factors. Therefore, the purpose of this study is to develop the health risk assessment index system for the left-behind elderly dependant on the health ecology model so that the health problems can be found earlier and the ability of assessing risk factors of elderly diseases can be improved. Thus, the health condition and quality of life of the left-behind elderly will be improved. Healthy ageing will be achieved easily.

\section{Materials and Methods}

The Delphi method was used, which was developed by Dalkey and Helmer in the 1950s [27]. It is an acknowledged method to gather consensus of opinion and choice about a topic [28]. The Delphi method is a structured iterative process that uses repetitive administration of questionnaires to gather information [29]. The Delphi method is widely used to develop assessment indicators in healthcare and achieve convergence opinions among experts and participants on specific topics [29,30]. The consensus process incorporated a two-round web-based Delphi method, which took place between July 2018 and November 2018.

\subsection{Experts Selection and Delphi Implemention}

Experts were selected to reflect the components of the health risk assessment. It is composed of geriatricians, health management experts, and social psychologists. There are no guidelines for the sample size of the Delphi study [31]. However, in general, the more panelists participate, the more reliable the group judgment will be [32]. Therefore, fifteen experts from six provinces and cities in China were selected. There was diverse geographical representation among the panel members, which came from various provinces of China including Beijing, Tianjin, Shanxi, Fujian, Zhejiang, and Jilin. The selection criteria were as follows: (1) working for 10 years and over in geriatrics, health management, social psychology, and other works, with rich professional theory and practical experience; (2) deputy senior and above technical titles; (3) informed consent and voluntary participation in this study. A key component of the Delphi technique is the anonymity of the expert panel members. Thus, none knew the identity of other panel members.

The informed consent for inclusion were given to all subjects before they participated in the study. The study was conducted in accordance with the Declaration of Helsinki, and the protocol was approved by the medical ethics committee of Tianjin University of Traditional Chinese Medicine.

The initial questionnaire with candidate indicators was pre-tested with three physicians (who were not recruited to the Delphi panel) to anticipate the average completion time and for clarity.

The Delphi questionnaires were sent out by e-mail. After summarizing and analyzing the experts' opinions in Round I, the addition, deletion, and modification of the previous round of questionnaire items were completed through a literature review and group discussion. The second round of expert correspondence was carried out. When the experts' opinions tended to be consistent, the correspondence ended.

\subsection{Questionnaire Preparation}

Based on the health ecology model, the five first-level indicators of the health risk assessment index system for the rural left-behind elderly were: personal characteristics, behavior characteristics, interpersonal network, living and working conditions, social, economic, cultural, and related policies. According to the definition of the first-level indicators, thirteen second-level indicators were determined.

Bibliography retrieval was conducted. "Old people, elderly, left behind, countryside, health, physical health, mental health, social adjustment" were used to search literature in English databases such as Web of Science, Pubmed, EMBASE, CINAHL and Chinese databases such as Wanfang Database, 
CNKI, and Weipu Database. On the basis of the literature research, health risk assessment indicators for the rural left-behind elderly were extracted. From this, an expert inquiry paper was compiled.

Through the retrieval of literature 4510 , deletion of duplicate literature 1326, remaining literature 3184 , reading topics and abstracts, 482 were screened, 234 full-text were read, 71 three-level indicators were extracted, which are shown in Figure 1.

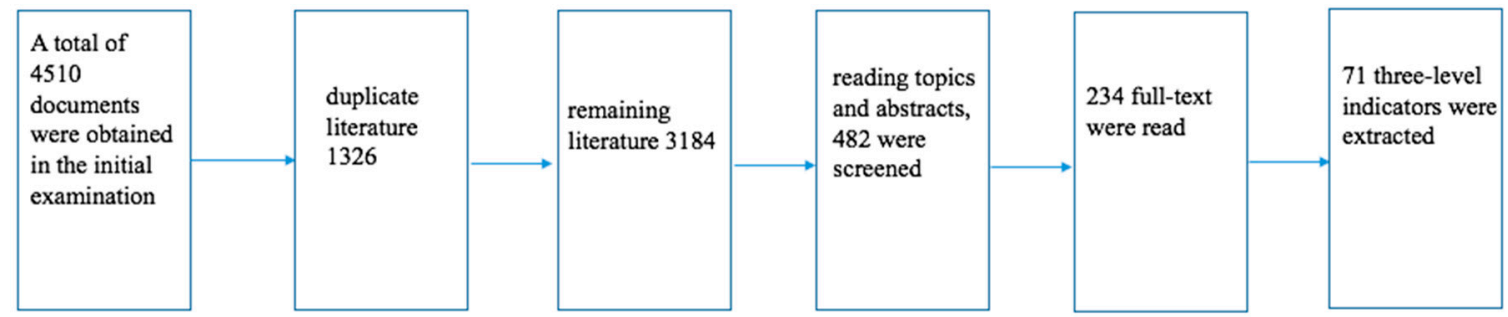

Figure 1. Steps taken to implement a bibliography retrieval.

A list of 71 indicators was included in the first-round questionnaire. These indicators were divided into personal traits, behavioral characteristics, interpersonal network, living conditions, social, economic, cultural, and related policies, which describe the health risks of the rural left-behind elderly. The first round of the expert inquiry included four parts: (1) the introduction of the subject, purpose, significance, and instructions of this Delphi; (2) basic information of experts, including age, position, title, length of service, work, and professional field, etc.; (3) expert opinion of the first-level, second-level, and third-level indicators of the health risk assessment for the rural left-behind elderly. Each indicator was accompanied by a column of importance judgment and revision comments for experts to put forward suggestions of the indicators. The Likert 5-level scoring method was adopted: "very important", "important", "general important", "unimportant", and "very unimportant" were assigned 5, 4, 3, 2, and 1 points, respectively. (4) Expert familiarity and judgment are used to understand the authority of the experts. Experts familiarity can be divided into five grades: very familiar, relatively familiar, generally familiar, a little familiar, and unfamiliar and assigned 0.9, 0.7, 0.5, 0.3, and 0.1, respectively. Expert judgment criteria are shown in Table 1.

Table 1. Expert judgment criteria.

\begin{tabular}{cccc}
\hline \multirow{2}{*}{ Judgment Basis } & \multicolumn{3}{c}{ Degree of Influence on Expert Judgment } \\
\cline { 2 - 4 } & Big & Medium & Small \\
\hline theoretical analysis & 0.1 & 0.1 & 0.1 \\
practical experience & 0.5 & 0.4 & 0.3 \\
peer understanding & 0.3 & 0.2 & 0.1 \\
intuitive perception & 0.1 & 0.1 & 0.1 \\
\hline
\end{tabular}

The indicators were revised according to the experts' opinions. In the second round of expert inquiry, experts were invited to re-comment the newly revised indicators. All experts who had participated in Round I were sent an email with the second-round questionnaires. But if the expert chose "know a little" and "not familiar" with the research content in the first round of the inquiry, the expert was not invited in the second round. The consensus of indicators in Round I, the modified and new indicators suggested by the experts in Round I were included in Round II [33]. The experts were asked to re-score each indicator using the same criteria based on their own opinion.

\subsection{Statistical Analysis}

The data were input by Excel (Microsoft Corporation, Redmond, WA, USA) and analyzed by SPSS17.0 (IBM Cor- poration, Chicago, IL, USA). The general information of experts is expressed by frequency and percentage; the measurement data are expressed by mean and standard deviation; 
the enthusiasm of experts is expressed by the rate of valid questionnaires (the number of returned questionnaires/the number of total questionnaires*100\%); and the coefficient of experts' authority $(\mathrm{Cr})$ is determined by the coefficient of experts' judgement (Ca) and experts' familiarity (Cs). The degree of concentration of experts' opinions is expressed by importance assignment mean (significance) and standard deviation (SD). The importance assignment mean significance $<3.5$ is taken as the criterion for deleting indicators. The coordination degree of experts' opinions is expressed by the coefficient of variation $(\mathrm{CV})$ and the coordination coefficient $(\mathrm{W}) . \mathrm{CV}>0.25$ is the criterion for deletion [34]. Yaahp software was used to calculate the weight of each index in the health risk evaluation index system of the rural left-behind elderly [35].

\section{Results}

\subsection{The Authority of Experts}

The characteristics of the experts who participated in the study are shown in Table 2. Fifteen experts were invited to participate in the Delphi study, thirteen (86.67\%) experts gave feedback in Round I, twelve (92.31\%) out of thirteen experts accepted the invitation and gave feedback in Round II.

Table 2. Main characteristics of the expert in two rounds of the Delphi study.

\begin{tabular}{ccc}
\hline Characteristics & $\begin{array}{c}\text { Experts in Round I } \\
(\boldsymbol{n}=\mathbf{1 3})\end{array}$ & $\begin{array}{c}\text { Experts in Round II } \\
(\boldsymbol{n}=\mathbf{1 2})\end{array}$ \\
\hline Age & $M=46.92, S D=5.63$ & $M=47.67, S D=5.18$ \\
\hline Gender & $2(15.38 \%)$ & $2(16.67 \%)$ \\
Male & $11(84.61)$ & $10(83.33 \%)$ \\
Female & $1(7.69 \%)$ & $1(8.33 \%)$ \\
\hline Province & $5(38.46 \%)$ & $4(33.33 \%)$ \\
Beijing & $1(7.69 \%)$ & $1(8.33 \%)$ \\
Tianjin & $3(23.08 \%)$ & $3(25 \%)$ \\
Shanxi & $2(15.38 \%)$ & $2(16.67 \%)$ \\
Fujian & $1(7.69 \%)$ & $1(8.33 \%)$ \\
Zhejiang & & $6(50 \%)$ \\
Jilin & $7(53.85 \%)$ & $4(33.33 \%)$ \\
Speciality & $4(30.77 \%)$ & $2(16.67 \%)$ \\
Geriatrics & $2(15.38 \%)$ & \\
Health management & & $5(41.27 \%)$ \\
Social psychology & $5(38.46 \%)$ & $7(58.33 \%)$ \\
\hline Professional title & $8(61.54 \%)$ & \\
Senior professional title & & \\
Sub-senior professional & & \\
title &
\end{tabular}

\subsection{The Authority of Experts}

The judgment criteria and familiarity of experts in the two-round Delphi study are shown in Tables 3 and 4. The judgment coefficient of experts is 0.87 . The expert familiarity is 0.76 . The authority coefficient of experts is 0.82 .

Table 3. Expert judgment criteria in Round I.

\begin{tabular}{ccccccc}
\hline \multirow{2}{*}{ Judgment Basis } & \multicolumn{2}{c}{ Big } & \multicolumn{2}{c}{ Medium } & \multicolumn{2}{c}{ Small } \\
\cline { 2 - 6 } & Number & Frequency & Number & Frequency & Number & Frequency \\
\hline Theoretical analysis & 11 & $84.62 \%$ & 2 & $15.38 \%$ & 0 & $0.00 \%$ \\
Practical experience & 8 & $61.54 \%$ & 5 & $38.46 \%$ & 0 & $0.00 \%$ \\
Peer understanding & 3 & $23.08 \%$ & 8 & $61.54 \%$ & 2 & $15.38 \%$ \\
Intuitive perception & 0 & $0.00 \%$ & 6 & $46.15 \%$ & 7 & $53.85 \%$ \\
\hline
\end{tabular}


Table 4. Expert familiarity in Round I and Round II.

\begin{tabular}{cccccc}
\hline Expert Familiarity & Very Familiar & Relatively Familiar & Generally Familiar & A Little Familiar & Unfamiliar \\
\hline Round I (Number of experts) & 6 & 5 & 2 & 0 & 0 \\
Round II (Number of experts) & 6 & 5 & 1 & 0 & 0 \\
\hline
\end{tabular}

\subsection{Delphi Round I}

In Delphi Round I, there are five first-level indicators including personal traits, behavioral characteristics, interpersonal network, living conditions, social, economic, cultural, and related policies. There are thirteen second-level indicators which includes native traits, disease susceptibility, psychosocial characteristics, habits, health behavior, family interpersonal network, community interpersonal network, social interpersonal network, conditions for medical treatment, socio-economic status, political environment, economic environment, and cultural environment. Seventy-one third-level health risk assessment indicators for the rural left-behind elderly were formed. The indicators reached consensus in Round I were included in the questionnaire of the Round II.

Combined with the statistical results and the suggestions of the experts in Delphi Round I, six three-level indicators were deleted, such as "the length of telephone conversation with children, the gender of children, the intensity of labor services", and eight three-level indicators were added, such as "children bear medical expenses", "social assistance", "cultural and recreational activities". At the same time, fifteen third-level indicators were modified, such as "daily living ability" was changed to "activities of daily living" and "alcoholism" was changed to "drinking".

\subsection{Delphi Round II}

After Delphi Round I, there are five first-level indicators, thirteen second-level indicators, and seventy-three third-level indicators in the health risk assessment index system. In Delphi Round II, experts have a high concentration on indicators at all levels, indicating that experts tend to agree. So, the correspondence ends. Combined with the expert opinions in Round II, after the literature review and group discussion, seven three-level indicators, such as "number of children", "frequency of communication with children", "family members as medical workers", were deleted. Finally, the health risk assessment index system for the rural left behind elderly was formed, which includes five first-level indexes, thirteen second-level indexes, and sixty-six third-level indexes. Two rounds of Delphi process are shown in Figure 2. Also, in Delphi Round II, the weight of indicators were given by experts.

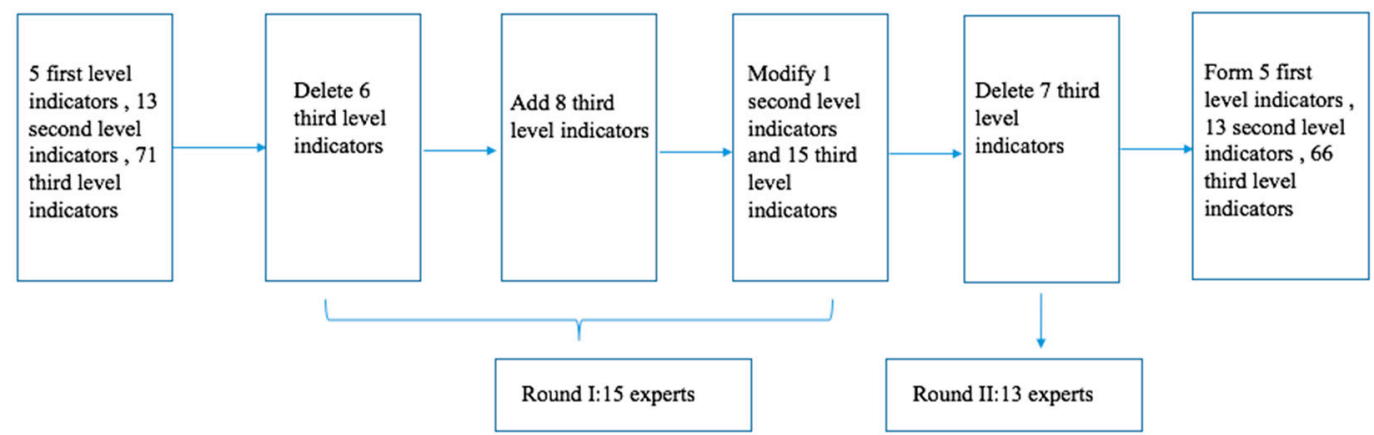

Figure 2. A Delphi study on the health risk assessment index system for the left-behind elderly.

\subsection{Final Indicators}

After the two-round Delphi study, five first-level indicators, thirteen second-level indicators, and sixty-six third-level indicators were compiled. The results are presented in Table 5. In the first-level indicators, personal traits $(0.248)$ and behavioral traits $(0.248)$ were the most important indicators. 
In the second-level indicators, the largest weight value is the medical condition (0.149). The largest weight value of the three-level indicators is the assistance provided by medical institutions (0.111).

Table 5. Indicators of the health risk assessment index system for the rural left-behind elderly.






\section{Discussion}

Guided by the health ecology model, the health risk assessment index system for the rural left-behind elderly was developed. The weights of the indicators indicate their importance [36]. Personal traits and behavioral traits were the most important first-level indicators in this study. At present, relevant studies have also confirmed that personal traits have impact on the health of the left-behind elderly. Glaesmer et al. surveyed 1659 elderly people in Germany, the results showed that age affects their mental health. The age of stay-at-home and education were related to their lower physiological and mental health level [14]. Regarding the behavioral characteristics, He et al. showed that the depression of 509 rural left-behind elderly was related to their physical activity ability [12]. Through the analysis of 209 left-behind elderly in Henan Province, Zhao and Zhang found that the quality of life of the rural left-behind elderly is affected by many factors, including age, negative life events, per capita annual income, social support, marriage, and family [37]. The second-level indicator with the largest weight value is the medical condition. Income level can affect the health status of the elderly. The elderly with different income have different medical behavior. The higher the income and the medical condition, the better their health condition [38]. As some researchers point out, children's migration contributes to the material well-being of their parents and better economic status is associated with less adverse health outcomes [7,39]. Some studies also show that heavy living burden and shortage of available medical service resources lead to higher incidence of stroke and heart disease for the left behind elderly. Also, when negative emotions such as loneliness increase, the mental health and self-rated health status reduce [21,39]. The three-level indicator with the largest weight value is the assistance provided by medical institutions. It indicates that the left-behind elderly trust the professional guidance given by medical institutions. So medical institutions, especially medical staff, should provide more medical and nursing services for the elderly. There is a significant positive correlation between the level of rural medical service and the objective health status of the rural left-behind elderly [40].

Valid and reliable measures depend on high-quality data [41]. This study used a series of scientific measures. But the Delphi method has its limitations, including purposeful selection of the panelists, attrition rate, and non-response bias although the two-round Delphi study and reminder letters helped to prevent attrition. A separate study is required to determine minimum data for implementing the measures [42]. Secondly, there were no left-behind elderly participating in the development process. The left-behind elderly with health problems should be invited in the development process in future research. Moreover, the study was anonymous, so we were unable to find changes between two rounds within respondents. Finally, the study investigators assumed that all the Delphi experts are familiar with the relevant knowledge of the research theme. However, it is conceivable that not all the experts were aware of the measures that reached agreement.

\section{Conclusions}

The Delphi technique has been used to develop the health evaluating indicators in previous studies. On the basis of literature research and expert inquiries, a health risk assessment index system for rural left-behind elderly was formed with five first-level indicators, thirteen second-level indicators, and sixty-six third-level indicators. The recommending indicators of this study are intended to provide a comprehensive tool to evaluate the health risk of the rural left-behind elderly. It also can be used for medical workers and health managers to identify the health risks of the rural left-behind elderly earlier. In particular, policy-makers can design future care systems for rural older adults. Therefore, the quality of life and health level of the rural left-behind elderly can be improved, the healthy ageing of the rural left-behind elderly can be promoted.

Author Contributions: Conceptualization, R.L. and C.Z.; methodology, R.L.; Software, R.L.; Validation, R.L., C.Z. and Y.L.; formal analysis, R.L.; investigation, R.L.; resources, Y.L.; data curation, Y.L.; writing一original draft preparation, R.L.; writing-review and editing, Y.L. and C.Z.; Visualization, Y.L.; Supervision, Y.L.; Project 
Administration, C.Z. Funding acquisition, C.Z. All authors have read and agreed to the published version of the manuscript.

Funding: This study was supported by "Study on Health Risk Assessment Model and Assessment Tool Construction for Rural Left-behind Elderly", which was grant from the National Natural Science Foundation of China (Project Approval Number: 71704133).

Acknowledgments: The authors would like to acknowledge the experts who took part in the Delphi Rounds 1 and 2.

Conflicts of Interest: The authors declare no conflict of interest. The funders had no role in the design of the study; in the collection, analyses, or interpretation of data; in the writing of the manuscript, or in the decision to publish the results.

\section{References}

1. Cao, G.Z.; Bian, X.; Zhao, J.H. The structural character and spatial disparity of left-behind families in rural China: Evidence from a nationwide survey in 30 counties in 6 provinces. Popul. Dev. 2013, 19, 2-10.

2. Zhou, L.; Wang, G.; Jia, C. Being left-behind, mental disorder, and elderly suicide in rural China: A case-control psychological autopsy study. Psychol. Med. 2018, 49, 1-7. [CrossRef]

3. Ye, J.Z.; He, Z.C. The Silent Sunset: The Left-Behind Elderly in Rural China Areas; Beijing Social Science Press: Beijing, China, 2008.

4. Carballo, M.; Divino, J.J.; Zeric, D. Migration and health in the European Union. Trop. Med. Int. Health 1998, 3, 936-944. [CrossRef] [PubMed]

5. Federal Interagency Forum on Aging-Related Statistics. Older Americans 2012: Key Indicators of Well-Being. Federal Interagency Forum on Aging-Related Statistics; Government Printing Office: Washington, DC, USA, 2012.

6. Theeke, L.A. Predictors of loneliness in U.S. adults over age sixty-five. Arch. Psychiatr. Nurs. 2009, 23, 387-396. [CrossRef] [PubMed]

7. Li, L.W.; Liu, J.; Xu, H.; Zhang, Z. Understanding rural-urban differences in depressive symptoms among older adults in china. J. Aging Health 2016, 28, 341-362. [CrossRef] [PubMed]

8. Song, Q. Facing double jeopardy? Depressive symptoms in left-behind elderly in rural china. J. Aging Health 2017, 29, 1182-1213. [PubMed]

9. Yang, X.L.; Xu, H.; Tang, G.Z.; He, Z.C.; Tu, D.; Yang, Y. Two-week prevalence rate and risk factors of rural left-behind elderly in Chongqing. Chongqing Med. 2015, 44, 2947-2948.

10. Abas, M.; Tangchonlatip, K.; Punpuing, S.; Jirapramukpitak, T.; Darawuttimaprakorn, N.; Prince, M.; Flach, C. Migration of Children and Impact on Depression in Older Parents in Rural Thailand, Southeast Asia. JAMA Psychiatry 2013, 70, 226-234. [CrossRef]

11. Antman, F.M. Adult Child Migration and the Health of Elderly Parents Left Behind in Mexico. Am. Econ. Rev. 2010, 100, 205-208. [CrossRef]

12. He, G.; Xie, J.F.; Zhou, J.D.; Zhong, Z.Q.; Qin, C.X.; Ding, S.Q. Depression in left-behind elderly in rural china: Prevalence and associated factors. Geriatr. Gerontol. Int. 2016, 16, 638-643. [CrossRef]

13. Miltiades, H.B. The social and psychological effect of an adult child's emigration on non-immigrant Asian Indian elderly parents. J. Cross Cult. Gerontol. 2002, 17, 33. [CrossRef] [PubMed]

14. Glaesmer, H.; Riedel-Heller, S.; Braehler, E.; Spangenberg, L.; Luppa, M. Age-and gender-specific prevalence and risk factors for depressive symptoms in the elderly: A population-based study. Int. Psychogeriatr. 2011, 23, 1294-1300. [CrossRef] [PubMed]

15. Ding, Z.J.; Wang, G.P.; Zhang, Y.L.; Xie, R.; Pei, G.X.; Du, H.Y. Epidemiological survey of mental disorders in the rural left behind elderly aged 60 years and older in Gansu. Zhonghua Yi Xue Za Zhi 2019, 99, 2429-2434. [PubMed]

16. Forlani, C.; Morri, M.; Ferrari, B.; Dalmonte, E.; Menchetti, M.; De Ronchi, D.; Atti, A.R. Prevalence and gender differences in late-life depression: A population-based study. Am. J. Geriatr. Psychiatry 2014, 22, 370-380. [CrossRef] [PubMed]

17. Houtjes, W.; Deeg, D.; Van de Van, P.; Van Meijel, B.; Van Tilburg, T.; Beekman, A. Is the naturalistic course of depression in older people related to received support over time? results from a longitudinal population-based study. Int. J. Geriatr. Psychiatry 2017, 32, 657-663. [CrossRef] 
18. Williams, L.; Zhang, R.; Packard, K.C. Factors affecting the physical and mental health of older adults in china: The importance of marital status, child proximity, and gender. SSM Popul. Health 2017, 3, $20-36$. [CrossRef]

19. Zhong, Y.; Pär, S.; Bo, B.; Kristina, B. Association between social capital and health-related quality of life among left behind and not left behind older people in rural china. BMC Geriatr. 2017, 17, 287. [CrossRef]

20. Xie, J.F.; Ding, S.Q.; Zhong, Z.Q.; Yi, Q.F.; Zeng, S.N.; Hu, J.H.; Zhou, J.D. Mental health is the most important factor influencing quality of life in elderly left behind when families migrate out of rural China. Rev. Lat. Am. Enferm. 2014, 22, 364-370. [CrossRef]

21. Adhikari, R.; Jampaklay, A.; Chamratrithirong, A. Impact of children's migration on health and health care-seeking behavior of elderly left behind. BMC Public Health 2011, 11, 143. [CrossRef]

22. Fu, H. Preventive Medicine; People's Health Press: Beijing, China, 2008.

23. Fisher, E.B.; Brownson, C.A.; O'toole, M.L. Ecological approaches to self-management: The case of diabetes. Am. J. Public Health 2005, 95, 1523-1535. [CrossRef]

24. Huberty, J.L.; Balluff, M.; O'dell, M. From good ideas to actions: A model-driven community collaborative to prevent childhood obesity. Prev. Med. 2010, 50, S36. [CrossRef] [PubMed]

25. Maibach, E.; Abroms, L.; Marosits, M. Communication and Marketing as Tools to Cultivate the Public's Health. BMC Public Health 2007, 7, 88. [CrossRef] [PubMed]

26. Anand, M. A Common Monitoring \& Evaluation Framework Guided by the Collective Impact Model: Recommendations to Enhance the Tobacco Control Effort in Sub-Saharan Africa. Master's Thesis, Georgia State University, Atlanta, GA, USA, 2013.

27. Dalkey, N.; Helmer, O. An experimental application of the Delphi method to the use of experts. Manag. Sci. 1963, 9, 458-467. [CrossRef]

28. Ryan, M.; Scott, D.A.; Reeves, C.; Bate, A.; van Teijlingen, E.R.; Russell, E.M.; Napper, M.; Robb, C.M. Eliciting public preferences for healthcare: A systematic review of techniques. Health Technol. Assess. 2001, 5, 1-186. [CrossRef]

29. Hsu, C.-C.; Sandford, B.A. The Delphi technique: Making sense of consensus. Pract. Assess. Res. Eval. 2007, 12, 1-8.

30. Boulkedid, R.; Abdoul, H.; Loustau, M.; Sibony, O.; Alberti, C. Using and reporting the Delphi method for selecting healthcare quality indicators: A systematic review. PLOS ONE 2011, 6, e20476. [CrossRef]

31. Prinsen, C.A.; Vohra, S.; Rose, M.R. Core Outcome Measures in Effectiveness Trials (COMET) initiative: Protocol for an international Delphi study to achieve consensus on how to select outcome measurement instruments for outcomes included in a 'core outcome set'. Trials 2014, 15, 247. [CrossRef]

32. Murphy, M.K.; Black, N.A.; Lamping, D.L. Consensus development methods, and their use in clinical guideline development. Health Technol. Assess. 1998, 2, 1-88. [CrossRef]

33. Young, J.M.; Masya, L.M.; Solomon, M.J.; Shepherd, H.L. Identifying indicators of colorectal cancer care coordination: A Delphi study. Colorectal Dis. 2014, 16, 17-25. [CrossRef]

34. Mcgeoch, M.; Brunetto, Y.; Brown, K. The policy delphi method: Contribution to policy and strategy within energy organisations: A 2013 malaysian case study with global implications. Qual. Quant. 2014, 48, 3195-3208. [CrossRef]

35. Rees, T. St john health, integrating new corporate identity, brand. brand/logo to be rolled out over two-year period. Profiles Healthc. Mark. 2003, 19, 14-18.

36. Pi, H.Y.; Peng, P.P.; Su, Q.Q.; Wang, Y.L.; Tang, J.P.; Zhang, J. A study on long-term care of quality evaluation index system for disabled elderly. Chin. J. Nurs. 2018, 53, 1110-1114.

37. Zhao, Y.X.; Zhang, H. Survey on the quality of life of the left-behind elderly in a rural area of Henan Province and analysis of its influencing factors. China Rural Health 2016, 13, 46-48.

38. Qiu, H.X.; Luo, W.; Xiao, Y.; Huang, J.Y. Comparison of health status and outpatient treatment behaviour of elderly people with different income levels. Chin. J. Gerontol. 2017, 37, 6225-6227.

39. He, C.; Ye, J. Lonely sunsets: Impacts of rural-urban migration on the left-behind elderly in rural China. Popul. Space Place 2014, 20, 352-369. [CrossRef]

40. Yang, S. Structural analysis of health evaluation and influencing factors of the old left behind in rural areas. J. Hebei Univ. Philos. Soc. Sci. 2017, 42, 154-160. 
41. Wakai, A.; O'Sullivan, R.; Staunton, P.; Walsh, C.; Hickey, F.; Plunkett, P.K. Development of key performance indicators for emergency departments in Ireland using an electronic modified-Delphi consensus approach. Eur. J. Emerg. Med. 2013, 20, 109-114. [CrossRef]

42. Buxton, M.J. Economic evaluation and decision making in the UK. Pharm. Econ. 2006, 24, $1133-1142$. [CrossRef]

(C) 2020 by the authors. Licensee MDPI, Basel, Switzerland. This article is an open access article distributed under the terms and conditions of the Creative Commons Attribution (CC BY) license (http://creativecommons.org/licenses/by/4.0/). 